\title{
ICP Registration Technology based on the Coordinate System Direction Fit
}

\author{
Heqiang Tian ${ }^{1}$, Peng $\mathrm{Yang}^{1}$, Chunjian $\mathrm{Su}^{1}$ and Zhiqiang Dong ${ }^{2}$ \\ College of Mechanical and Electronic Engineering, \\ Shandong University of Science and Technology, Qingdao266590, China \\ ${ }^{1}$ College of Mechanical and Electronic Engineering, \\ Shandong University of Science and Technology, Qingdao266590, China \\ ${ }^{2}$ West coast (Development) Group Co., Ltd of Qingdao, Qingdao 266590, China \\ thq_1980@126.com
}

\begin{abstract}
In computer assisted surgery, one of the most important problems is to align the preoperative model with intraoperative data. The Iterative Closest Point (ICP) algorithm is undoubtedly the most popular algorithm for solving this kind of problem, which needs a good initialization. In this paper, an ICP algorithm based on the coordinate system direction fit is present to improve ICP algorithm's shortcoming with easily falling into local optimum for intraoperative registration. Firstly, ICP registration algorithm and its problems are introduced. Secondly, based on the consistency between CT scans pose and surgical pose, a three-plane coordinate system on patients is established to reduce the rotation dislocation between CT image space and patient space. Then, an ICP algorithm experiment and its precision analysis are conducted. Finally, the error analysis is also given. The experimental results show that one of the benefits of the ICP algorithm based on the coordinate system direction fit is that it is easily convergent on the registration by a three-plane coordinate system on patients, which increases the registration accuracy and reduces the computational processing time.
\end{abstract}

Keywords: Intraoperative registration; ICP algorithm; Coordinate direction fit; Threeplane coordinate system

\section{Introduction}

In computer assisted surgery, one of the most important problems is to align the preoperative model with intraoperative data for surgical navigation. Mathematically, this is a problem of estimating the coordinate transformations, usually involving rotation and translation, between the two coordinate systems in which the preoperative data and intraoperative data are presented. Some feature points can be easily taken by using the probe tool of optical tracking system during the surgery procedure. Therefore, the pointbased registration method and surface-based registration method are more suitable for surgical navigation based on optical positioning. Various kinds of surface-based registration methods and point-based registration methods have been proposed to find the optimal transformation in surface models or point sets taken at different positions and orientations, for example, the surface-based registration used to evaluate the treatment outcomes in oral and maxillofacial surgery [1] and the point-based rigid registration used to plan orthognathic and facial orthomorphic surgery by computing the rotation and translation from the surface information and searching the corresponding points and shapes [2]. To overcome the multiple point correspondence problems of conventional surface registration methods, a novel depth map-based registration method to register 3D 
surface models of partially scanned dental surfaces in a 3D dental laser scanning was proposed [3].

Iterative closest point (ICP), proposed by Besl et al. [4], is one of the best known fitting or registration algorithms between 3D point clouds and has been widely used for several applications, including 3-D model fitting, shape registration, and human motion tracking. ICP (Iterative Closest Point) algorithm has good results for the point set registration problem without exact correspondence. However, it is well known that ICP requires a good initial pose and also an initial guess of the scale. ICP usually fails if the two point clouds differ in pose and scale. ICP algorithm has been improved in the algorithm level by many scholars at home and abroad. Greenspan and Yurick [5] proposed the approximate K-D tree that did not use expensive backtracking, but required an additional time to construct the K-D tree. Choi et al. [6] proposed the improved K-D trees traversal method to accelerate finding process of the nearest points. Benjemaa and Schmitt [7] proposed a multi z-buffer technique to perform the local search but required the acquisition geometry and calibration parameters. Daehwan Kim and Daijin Kim [8] proposed a fast ICP algorithm that uses a hierarchical model point selection and a 2-D logarithmic data point search to reduce corresponding closest data point search time. Minh-Due Tran et al. [9] proposed a method to select the best sample points to speed up the ICP algorithm, whose most important advantage is reducing the computation time and hence increasing real-time of system. Although these methods reduced the computation time required to find the corresponding closest points according to the rate of the sampling, the registration results provided the high error values. Pechenin et al. [10] proposed and confirmed the method of ICP algorithm accuracy improvement based on the solution of a multi-objective optimization problem used for identification of location deviation parameters for profiles and surfaces with shaped form of surface. Baowei Lin, et al.[11] proposed a scale alignment method of 3D point clouds with different scales which using a concept similar to ICP for directly estimating the scale ratio of two point clouds ,which is used to estimate the scale ratio for ICP methods. The proposed method works well both for simple and difficult point cloud datasets. But, the computational for generating spin images from the point clouds and computing PCA is increased.

The registration process usually includes two steps such as initial registration and accurate registration. The initial registration is used to reduce the rotational and translational misalignment between two object point sets in order to improve the accurate registration efficiency and trends, and the accurate registration minimizes the registration error between the two object point sets. For example, Xue Mei et al. [12] developed an improved Iterative Closest Point (ICP) algorithm that focuses on the registration of Cone Beam Computed Tomography (CT) image and high-resolution Blue-light scanner image, which includes two major phases, coarse and precise registration and has successfully realized the registration of the Cone Beam CT dental model and the blue-ray scanner model with higher accuracy. But when the two object point sets come to have the larger rotational displacement, the correct registration result is not obtained.

This paper presents an ICP algorithm based on the coordinate system direction fit which reduces rotation dislocation between $\mathrm{CT}$ image space and patient space to acquire registration matrix between two spaces to solve the local convergence problem for the best registration effect. This paper is organized as follows. Section 2 briefly reviews the existing ICP algorithm. Section 3 explains ICP registration technology based on the coordinate system direction fit. Section 4 introduces the ICP algorithm registration experiment and evaluates registration accuracy of the proposed method including the error analysis of the ICP experimental results. Finally, Section 5 presents our conclusion. 


\section{Iterative Closest Point Algorithm}

Besl and McKay developed ICP (Iterative Closest Point) algorithm based on the Berthold algorithm. Compared with point registration method, the biggest advancement of ICP algorithm does not need to point out the exact correspondence between the two point sets, but starts the iteration by an initial value. When the ICP algorithm is used for two sets registration, Delaunay tessellation corresponding point search method will be adopted to speed up the point search speed and improve the registration efficiency, which partitions the corresponding point sets into a group of simplex that is constituted by four points, and find the nearest point by starting from the simplex to greatly improve computational efficiency. After finding the corresponding point sets, the unit quaternion optimized analytical method will be used to calculate the optimal solution registration parameters between two sets.

For the registration between CT image space and patients space, the data set in patients space $W=\left\{w_{i}, i=0,1,2, \cdots, n\right\}$ and the data set in image space $M=\left\{m_{j}, j=0,1,2, \ldots, k\right\}$ are defined, where $n, k$ is the number of registration points and $k \geq n$, and where there is not necessarily one to one relationship between the elements in the data set $W$ and the elements in the data set $M$, also do not have the same number of elements. ICP registration algorithm is described as follows:

1) Read two point sets to be registered. Firstly, get the point set in the patient coordinates $W=\left\{w_{i}, i=0,1,2, \cdots, n\right\}$ and the point set in the image coordinates $M=\left\{m_{j}, j=0,1,2, \ldots, k\right\}$, then read these two registration point sets for the input data of ICP registration algorithm;

2) Calculate the nearest point. Use the Delaunay triangulation to search the nearest corresponding points from the data set $M$ for each point in the data set $W$, where the new point set composed by the corresponding points is defined as $M^{\prime}=\left\{m_{r}^{\prime}, r=0,1,2, \ldots, n\right\}$;

3) Calculate registration transformation matrix. After finding the corresponding points, use the unit quaternion optimization analytic method to calculate the transformation relationship between the data set $W$ and $M$, so as to get registration transformation matrix $R, T$ by $\min \sum_{s=1}^{n}\left\|m_{s}^{\prime}-\left(R\left(w_{s}\right)+T\right)\right\|^{2}$, where $R$ is a $3 \times 3$ rotation matrix and $T$ is a $3 \times 1$ translation matrix;

4) Update the coordinate point sets. Use the matrix $R, T$ to transform the point set $W$ to obtain the new point set $W^{\prime}=\left\{w_{t}^{\prime}, t=0,1,2, \cdots, n\right\}$, where $w_{t}^{\prime}=R\left(w_{t}\right)+T$;

5) Calculate the root mean square error. Calculate the RMSE between the point set $W$ and $M^{\prime}$. If the RMSE is less than the preset limit value $\varepsilon$, the iteration will terminate.

ICP algorithm requires a higher initial value. If the rotational and translational misalignment between the two point sets is too large, the accuracy and convergence rate of ICP algorithm will be affected and it may even fall into local optima. This fatal flaw would seriously affect the registration efficiency and accuracy between Image space and Patient space.

\section{Direction Fit Coordinate System Set}

Before performing cervical surgery, a patient's cervical CT images need to be taken firstly. CT images are stored in DICOM format, which is standard dedicated storage format for medical images. Currently, the coordinate axes in DICOM 3.0 are entirely the patient-centered right-handed coordinate system, which has LPS (left-posterior-superior) coordinate system whose coordinate axis direction is determined based on the patient's 
direction, where $\mathrm{X}$ axis points left hand side of the patient, $\mathrm{Y}$ axis points the back of the patient, and $\mathrm{Z}$ axis points the patient's head, as shown in Figure 1. The optical positioning device has its own coordinate system. The probe tool of an optical locator is used to obtain the point sets in the cervical spine surface, whose coordinate system is the coordinate system of the optical positioning device. The registration goal by the use of the optical positioning device is to obtain the translation and rotation transformation between the optical measuring spatial coordinate system and CT image space coordinate system. The larger direction dislocation between the optical measuring space coordinate system and the CT image space coordinate system will make ICP algorithm not to converge correctly. Therefore, a patient coordinate system with the orientation consistent with the CT images coordinate system can greatly reduce the direction dislocation between the CT image space and patient space, so as to improve the efficiency and precision of ICP algorithm, when the point sets in optical space transform to patient space.

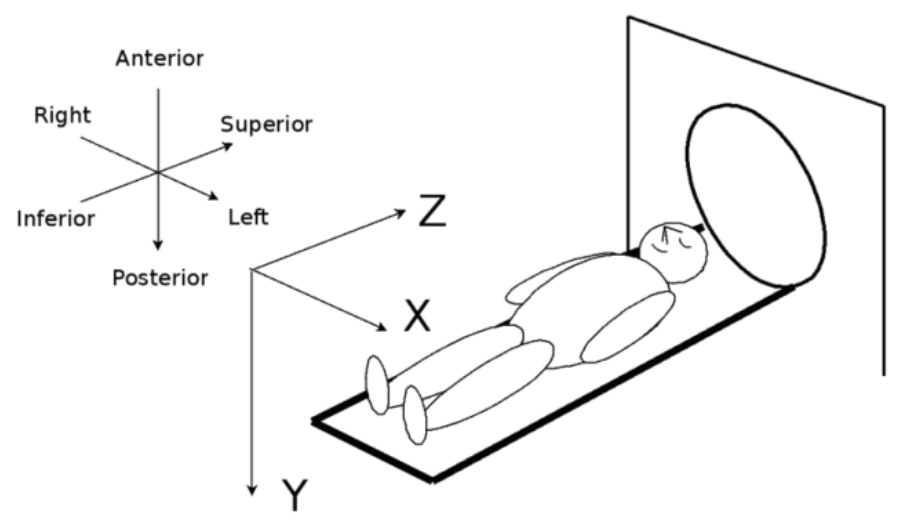

Figure 1. CT Coordinates System and Patient's Position and Pose

Direction fit coordinate system is established as follows: establish a patient coordinate system on the cube, and a optical tracking passive rigid body is mounted on the cubes in order to facilitate measuring, which has a coordinate system $\{S\}$ shown in Figure 2 a .

The patient coordinate system is established on the cube as follows: Three planes such as $\alpha, \beta$ and $\gamma$ are established among which any two are intersecting. The normals of three planes are vertical to the corresponding planes whose directions are toward the outer and normal vectors are expressed as $\left(A_{\alpha}, B_{\alpha}, C_{\alpha}\right),\left(A_{\beta}, B_{\beta}, C_{\beta}\right)$ and $\left(A_{\gamma}, B_{\gamma}, C_{\gamma}\right)$ with the sign judged using the optical locator as shown in Figure $2 \mathrm{~b}$. The patient coordinate system on the cube is established with the intersection $P$ of three planes defined as the origin, the line of intersection between the plane $\alpha$ and $\beta$ defined as $x_{p}$-axis, the opposite direction of the plane $\alpha$ normal vector defined as $y_{p}$-axis, and $z_{p}$-axis established according to the right-hand rule. This coordinate system's reference is only $\alpha$ plane. In theory, the plane $\alpha, \beta$ and $\gamma$ are not necessarily perpendicular each other. So, the coordinate system establishment will not bring new error. 


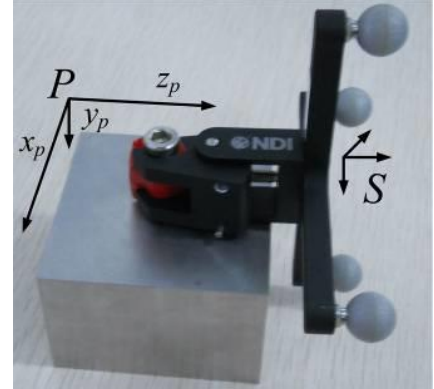

a) Cube coordinate system

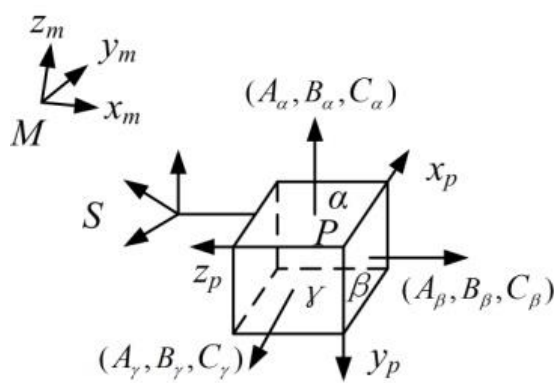

b) Three planes coordinate system

Figure 2. Patient Coordinate System Definition

The optical measurement coordinate system is defined as $M\left\{x_{m}, y_{m}, z_{m}\right\}$. Less than three non-collinear points can be obtained in the corresponding plane using an optical probe, which are used to define the plane equation of $\alpha, \beta$ and $\gamma$ in the optical measurement coordinate system as follows:

$A_{\alpha}\left(x-x_{\alpha}\right)+B_{\alpha}\left(y-y_{\alpha}\right)+C_{\alpha}\left(z-z_{\alpha}\right)=0$

$A_{\beta}\left(x-x_{\beta}\right)+B_{\beta}\left(y-y_{\beta}\right)+C_{\beta}\left(z-z_{\beta}\right)=0$

$A_{\gamma}\left(x-x_{\gamma}\right)+B_{\gamma}\left(y-y_{\gamma}\right)+C_{\gamma}\left(z-z_{\gamma}\right)=0$

The parameters such as $A_{i}, B_{i}, C_{i}, x_{i}, y_{i}$ and $z_{i}(i=\alpha, \beta, \gamma)$ can be obtained by detecting, and the symbol of $A_{i}, B_{i}$ and $C_{i}$ is defined by the optical locator. The point $P\left(x_{m p}, y_{m p}, z_{m p}\right)$ and linear equations for each coordinate axis can be solved in the optical measurement coordinate system $M\left\{x_{m}, y_{m}, z_{m}\right\}$, which are calculated as follows:

$$
\left[\begin{array}{l}
x_{m p} \\
y_{m p} \\
z_{m p}
\end{array}\right]=R^{-1}\left[\begin{array}{l}
x_{0} \\
y_{0} \\
z_{0}
\end{array}\right]
$$

Where

$$
\begin{gathered}
R=\left[\begin{array}{ccc}
A_{\alpha} & B_{\alpha} & C_{\alpha} \\
A_{\beta} & B_{\beta} & C_{\beta} \\
A_{\gamma} & B_{\gamma} & C_{\gamma}
\end{array}\right], \\
\\
\\
\\
z_{0}=A_{\gamma} x_{\gamma}+B_{\gamma} y_{\gamma}+C_{\gamma} z_{\gamma} . \\
y_{0}=A_{\alpha} x_{\alpha}+B_{\alpha} y_{\alpha}+C_{\alpha} z_{\alpha},
\end{gathered}
$$

$x_{p}$-axis equation:

$\frac{\left(x-x_{m p}\right)}{l_{x}}=\frac{\left(y-y_{m p}\right)}{m_{x}}=\frac{\left(z-z_{m p}\right)}{n_{x}}$,

$y_{p}$-axis equation:

$\frac{\left(x-x_{m p}\right)}{-A_{\alpha}}=\frac{\left(y-y_{m p}\right)}{-B_{\alpha}}=\frac{\left(z-z_{m p}\right)}{-C_{\alpha}}$,

$z_{p}$-axis equation: 


$$
\frac{\left(x-x_{m p}\right)}{l_{z}}=\frac{\left(y-y_{m p}\right)}{m_{z}}=\frac{\left(z-z_{m p}\right)}{n_{z}},
$$

Where

$$
l_{x}=\left|\begin{array}{ll}
B_{\alpha} & C_{\alpha} \\
B_{\beta} & C_{\beta}
\end{array}\right|, m_{x}=\left|\begin{array}{cc}
C_{\alpha} & A_{\alpha} \\
C_{\beta} & A_{\beta}
\end{array}\right|, n_{x}=\left|\begin{array}{cc}
A_{\alpha} & B_{\alpha} \\
A_{\beta} & B_{\beta}
\end{array}\right|,
$$

$l_{z}=\left|\begin{array}{cc}m_{x} & n_{x} \\ -B_{\alpha} & -C_{\alpha}\end{array}\right|, m_{z}=\left|\begin{array}{cc}n_{x} & l_{x} \\ -C_{\alpha} & -A_{\alpha}\end{array}\right|, n_{z}=\left|\begin{array}{cc}l_{x} & m_{x} \\ -A_{\alpha} & -B_{\alpha}\end{array}\right|$.

The homogeneous coordinate transformation matrix ${ }^{M} T_{P}$ can be obtained by the following formula according to the origin of the coordinate system $P\left\{x_{p}, y_{p}, z_{p}\right\}$ and the three coordinate axes equations.

$$
{ }^{M} T_{P}=\left[\begin{array}{cccc}
\frac{l_{x}}{L_{x}} & \frac{-A_{\alpha}}{L_{y}} & \frac{l_{z}}{L_{z}} & x_{m p} \\
\frac{m_{x}}{L_{x}} & \frac{-B_{\alpha}}{L_{y}} & \frac{m_{z}}{L_{z}} & y_{m p} \\
\frac{n_{x}}{L_{x}} & \frac{-C_{\alpha}}{L_{y}} & \frac{n_{z}}{L_{z}} & z_{m p} \\
0 & 0 & 0 & 1
\end{array}\right]
$$

Where

$$
L_{x}=\sqrt{l_{x}^{2}+m_{x}^{2}+n_{x}^{2}}, L_{y}=\sqrt{A_{\alpha}^{2}+B_{\alpha}^{2}+C_{\alpha}^{2}}, \quad L_{z}=\sqrt{l_{z}^{2}+m_{z}^{2}+n_{z}^{2}} \text {. }
$$

The transformation matrix ${ }^{M} T_{S}$ can be obtained directly on the passive rigid body coordinate from the passive rigid body coordinate system $\{S\}$ on the cubes to the optical measurement coordinate system $\{M\}$ by optical locator, and then the conversion relationship ${ }^{5} T_{P}$ from the patient coordinate system to the optical measurement coordinate system can be also obtained. As shown in Figure 3, the cube is fixed together with the operating table in the surgical procedure so as to have a near-unanimous rotation between $\mathrm{CT}$ coordinate system and patient coordinate system defined on the cube, which is possible to transform the point set of the cervical vertebrae surface obtained by probe to the patient coordinate system, so as to ${ }^{V} T_{P}$ between the CT image space and the patient space can be achieved using the ICP algorithm. ${ }^{s} T_{P}$ can be obtained by the formula as follows:

$$
{ }^{S} T_{P}=\left({ }^{M} T_{S}\right)^{-1 M} T_{P}
$$




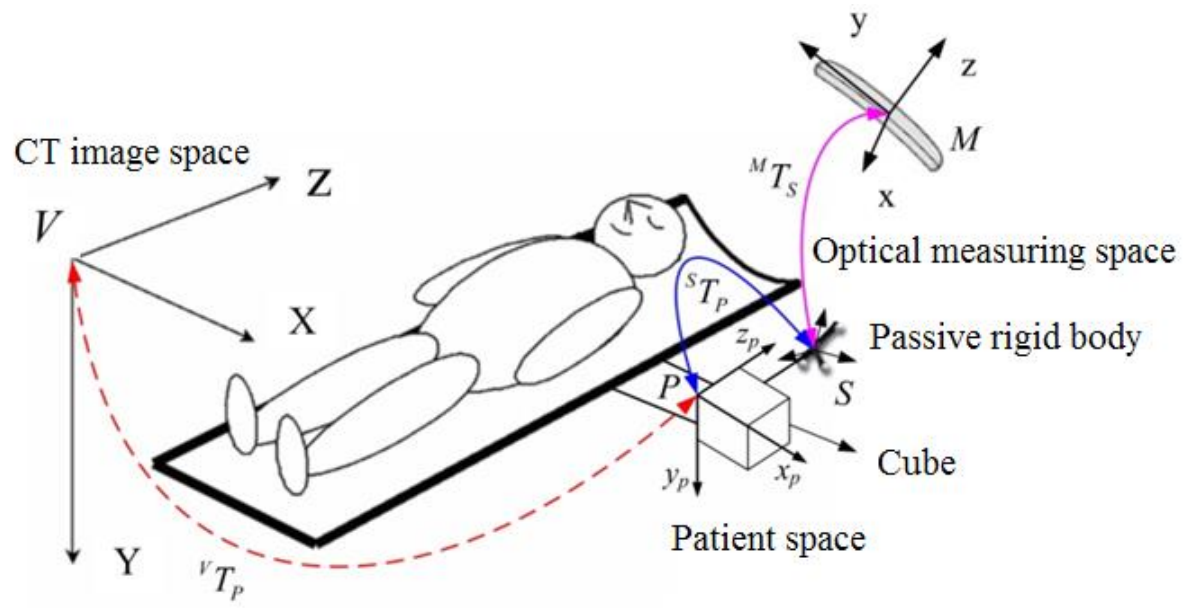

Figure 3. Position Relationship between CT Image Space and Patient Space

\section{ICP Algorithm Registration Test}

\subsection{ICP Registration Example}

A spine model is scanned by CT with the scanned image layer spacing set for $0.625 \mathrm{~mm}$ and the pixel pitch for $0.439 \mathrm{~mm}$. 3D surface model of the spine model which is used in the ICP algorithm registration test is reconstructed by using MC method. Three distinct features of the 3D model are selected as the registration area, as shown in Figure 5. The registration point sets in the 3D model registration feature area are obtained by the use of an interactive method developed using VTK visualization toolkit, which can complete digital acquisition of the feature surfaces in image space.

In order to realize digital capture of the feature surfaces in surgical space, the optical locator probe tool is used to real-time pick the registration point sets in surgery space on the feature surfaces corresponding to the model surface features. In order to obtain point cloud data with their distribution more uniform and quality high, the appropriate sampling threshold is set in the data collection process. Due to the limitations of the surgical operative area and the different in the intraoperative surgical object exposure, the feature region selected in the actual surgical space is only a subset of the feature region selected in image space.

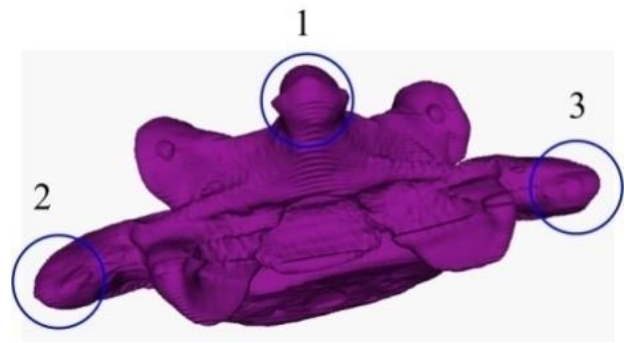

Figure 4. Three Registration Characteristic Zones

The registration point sets are acquired in the three feature regions as shown in Figure 4. 648 points in image space are obtained for registration point sets in the image space, and 577 points are collected by the use of the probe tool for registration point sets in the surgical space. ICP algorithm not be improved is directly used to register two point sets between the image space and the surgical space with the registration results shown in Figure 5. It can be seen that the registration result between two point sets shown in Figure 
6a is very poor and the two point sets shown in Figure $6 \mathrm{~b}$ have larger position and pose deviation. The main reason why the registration error is very big is that the two point sets between image space and surgical space have larger rotation dislocation, which can cause ICP algorithm not to converge correctly.

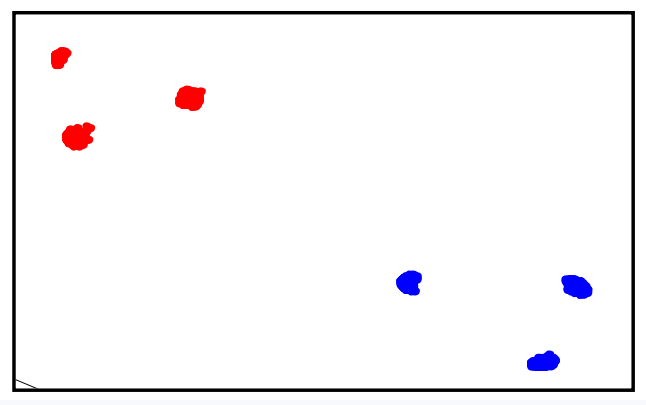

a) Non- registered

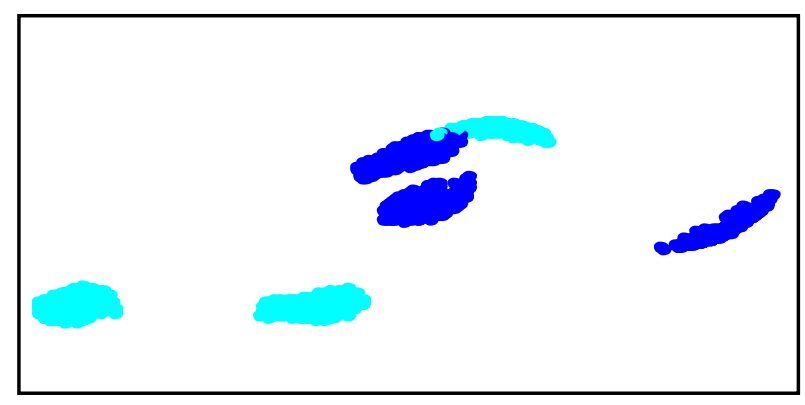

b) Be-registered

Figure 5. Registration Result between Image Space and Surgical Space

In order to solve the problem above, the point sets collected by the probe tool in optical measure space are transformed to the patient coordinate system established in the paper, which can be seen in Figure 6a that the two point sets in image space and patient space have closely consistent coordinate system rotation. Figure $6 \mathrm{~b}$ shows the very good registration result which is realized using ICP algorithm between image space and patient space. Thus, the registration test shows that ICP registration technology based on the coordinate system direction fit proposed in the paper used in registration between image space and patient space is very feasible which greatly improves the registration efficiency.

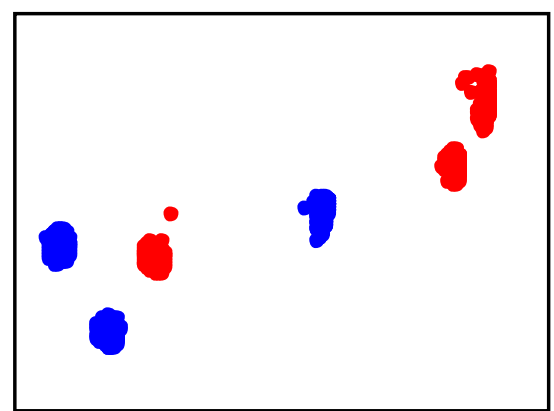

a) Non- registered

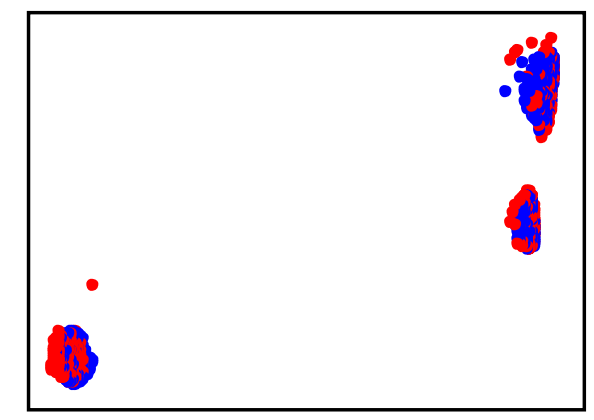

b) Be-registered

Figure 6. Registration Result between Image Space and Patient Space

\subsection{Accuracy Verification for ICP Registration}

It is easy for the metal ball to show shape in CT image. So, the metal ball can be used to check registration accuracy with 5 metal balls pasted on the spine model, as shown in Figure 7a. And the 3D reconstruction model with 5 metal balls of the spine model is shown in Figure $7 \mathrm{~b}$. Then the coordinate values of 5 metal balls in image space and patient space were obtained respectively. In order to avoid the effect of random errors, 10 sets of data for each ball were obtained, whose average value is defined as the coordinate value of each ball, as shown in Table 1 . The $\operatorname{dist}\left({ }^{v} P_{i},{ }^{T} P_{i}\right)$ is defined for the distance between the coordinate value ${ }^{v} P_{i}$ and ${ }^{T} P_{i}$, where ${ }^{v} P_{i}$ is the coordinate value of the $i$ th metal ball in image space and ${ }^{T} P_{i}$ is the coordinate 
value of the $i$ th metal ball obtained by ICP registration, and where $i=1,2 \cdots, 5$. The average registration error is defined as follows:

$$
E r=\frac{1}{5} \sum_{1}^{5}\left(\operatorname{dist}\left({ }^{v} P_{i},{ }^{T} P_{i}\right)\right)
$$

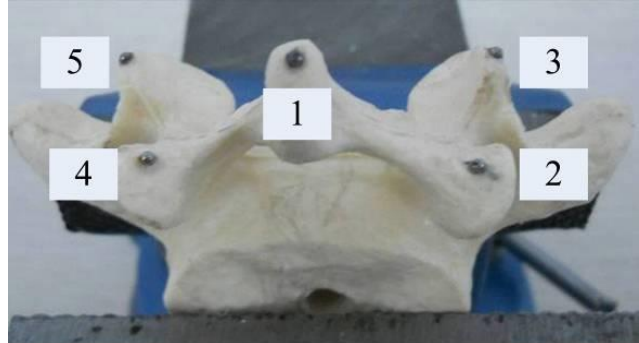

a) Spine Model

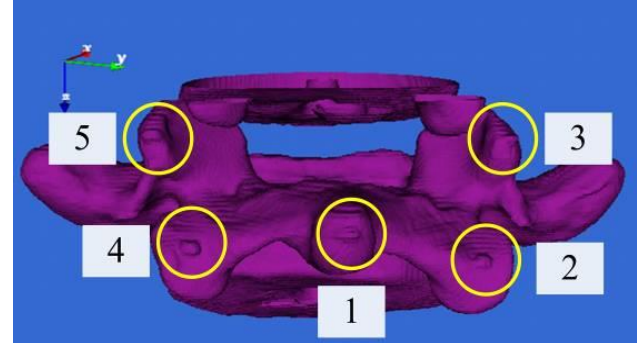

b) 3D Model

Figure 7. Spine Model with Five Balls and its 3D Model

Table 1. Registration Errors for Spine Model

\begin{tabular}{cccc}
\hline No. & $\begin{array}{c}\left(x_{V}, y_{V}, z_{V}\right)(\mathrm{mm}) \\
\text { in image space }\end{array}$ & $\begin{array}{c}\left(x_{P}, y_{P}, z_{P}\right)(\mathrm{mm}) \\
\text { in patient space }\end{array}$ & Average error(mm) \\
\hline 1 & $65.5378,142.965,15.975$ & $-35.9187,37.8178,-82.717$ & 1.32 \\
2 & $92.4492,152.344,25.122$ & $-5.0885,44.6129,-77.269$ & 1.67 \\
3 & $103.055,152.532,5.506$ & $-3.9934,45.6484,-98.408$ & 1.34 \\
4 & $69.3394,115.42,21.569$ & $-36.9055,8.7329,-82.080$ & 1.41 \\
5 & $71.8725,107.543,3.9335$ & $-43.5253,3.5188,-101.56$ & 1.54 \\
\hline
\end{tabular}

Ten ICP registration tests were carried on, whose average registration errors with the maximum average error expressed as $1.67 \mathrm{~mm}$ shown in Table 1 were calculated by the formula (7). Herring [13] pointed out that the body's bone surface changes greatly in curvature and the surface registration error for ICP algorithm may be lower than $2 \mathrm{~mm}$.

It can be seen that the registration transformation between image space and patient space by use of ICP algorithm is reliable and accurate. Registration accuracy is influenced by many factors such as image scanning accuracy, mode reconstruction accuracy, registration algorithm accuracy, positioning accuracy a, random error and so on. Layer spacing and resolution of preoperative scan slices image will affect the accuracy of threedimensional reconstruction. If the surface reconstruction model is closer to real object, the registration point coordinates obtained in the reconstruction model is more accurate and the registration error is smaller. The positioning precision of the overall system is determined by the locator. In addition, human factors are unpredictable and have a random nature, which will bring the some errors.

\section{Conclusions}

To solve the problem that ICP algorithm falls into local optimum easily, based on the patient's position and pose consistency for CT scan and surgery, ICP registration based on the coordinate system direction fit is proposed to reduce rotation dislocation between CT image space and patient space, which uses three planes to establish the patient coordinate system to achieve the intraoperative registration between image space and patient space. The test results show that this method solves the problems that the original algorithm requires a higher initial value, high computational complexity, and long registration time, 
which successfully establishes the spatial transformation between between image space and patient space and improves the efficiency and accuracy of surgery navigation .

\title{
Acknowledgments
}

The authors would like to express appreciation to financial supports from Shandong Province Young and Middle-Aged Scientists Research Awards Fund (BS2013ZZ011), Qingdao application foundation research project (Youth special) (14-2-4-120-jch), Scientific Research Foundation of Shandong University of Science and Technology for Recruited Talents (2013RCJJ016), National Natural Science Foundation of China (51305241) and Shandong province high school scientific research plan project (J12LA03)

\section{References}

[1] T.J. Maal, B. Loon, J.M. Plooij, F. Rangel, A.M. Ettema, W.A.Borstlap, S.J. Bergé, Registration of 3dimensional facial photographs for clinical use, J. Oral Maxillofac. Surg. 68 (10), pp.2391-2401(2010).

[2] G.R.J. Swennen, M.Y. Mommaerts, J. Abeloos, C.D. Clercq, P.Lamoral, N. Neyt, J. Casselman, F. Schutyser, A cone-beam CT based technique to augment the 3D virtual skull model with a detailed dental surface, Int. J. Oral Maxillofac. Surg. 38(1), pp.48-57(2009).

[3] S. Park, Ho Chul Kang, Jeongjin Lee, Juneseuk Shin, Yeong Gil Shin. An enhanced method for registration of dental surfaces partially scanned by a 3D dental laser scanning. Computer Methods and Programs in Biomedicine.118, pp.11-22(2015).

[4] P. Besl and N. McKay. A method for registration of 3-d shapes. IEEE Trans. Pattern Anal. Mach. Intell.. vol. 14 , no. 2, pp. 239-256(1992)

[5] M. Greenspan and M. Yurick. Approximate k-d tree search for efficient ICP. In Proc. Fourth Int. Conf. 3-D Digital Imaging and Modeling (3DIM).pp. 442-448(2003)

[6] W. S. Choi, Y. S. Kim, S. Y. Oh, and J. Lee. Fast iterative closest point framework for 3D LIDAR data in intelligent vehicle. In Proceedings of the IEEE Intelligent Vehicles Symposium. pp. 1029-1034, Madrid, Spain, (2012).

[7] Benjemaa and F. Schmitt. A fast global registration of 3D sampled surfaces using a multi-Z-buffer technique. In Proc. Int. Conf. Recent Advances in 3-D Digital Imaging and Modeling. pp. 113120(1997).

[8] D. Kim,Kim D.A fast ICP algorithm for 3-D human body motion tracking. Signal Processing Letters, IEEE. 17(4), pp. 402-405(2010).

[9] MD Tran, HJ Kang, YS Ro. Improved ICP control algorithm in robot surgery application. IFOST 2010 Proceedings. Ulsann, Korea: IEEE. pp. 1-4(2010).

[10] V.A. Pechenin, M.A. Bolotov, N.V. Ruzanov. Development of a method of ICP algorithm accuracy improvement during shaped profiles and surfaces control. International Journal of Engineering and Technology. Vol 6, No 5, pp.2229-2235, (2014).

[11] Baowei Lin,Toru Tamaki,Fangda Zhao,Bisser Raytchev,Kazufumi Kaneda,Koji Ichii. Scale alignment of 3D point clouds with different scales. Machine Vision and Applications. 25, pp.1989-2002(2014).

[12] XueMei, Z. Li, Songsong Xu, and Xiaoyan Guo. Registration of the Cone Beam CT and Blue-Ray Scanned Dental Model Based on the Improved ICP Algorithm. International Journal of Biomedical Imaging.pp.1-8 (2014).

[13] Herring, JL, Dawant, BM, Maurer, CR, Muratore, DM, Galloway, RL, and Fitzpatrick, JM. Surfacebased registration of CT images to physical space for image-guided surgery of the spine: a sensitivity study. IEEE Trans. Med. Imag.. 17(5), pp.743-752(1998).

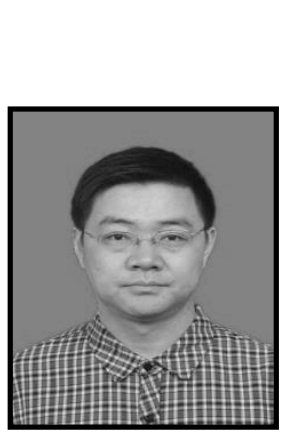

\begin{abstract}
Author
Heqiang Tian. Current position: He is lecturer of Mechanical and Electronic Engineering College, Shandong University of Science and Technology in China. University studies: Doctor of Mechanical and Electrical Engineering College from Harbin Institute of Technology in China. Scientific interest: Computer Assisted Surgery, Medical Robotics. Publications: More than 10 papers published in various journals. Experience: Teaching experience of 3 years, 5 scientific research projects.
\end{abstract}

